Cahiers $d u$ MONDE RUSSE

\section{Cahiers du monde russe}

Russie - Empire russe - Union soviétique et États indépendants

$61 / 3-4 \mid 2020$

Écritures visuelles, sonores et textuelles de la justice

Jan HENNINGS, Russia and Courtly. Europe Ritual and the Culture of Diplomacy, 1648-1725

\title{
Aleksandr Lavrov
}

\section{OpenEdition}

\section{Journals}

Édition électronique

URL : https://journals.openedition.org/monderusse/12081

DOI : $10.4000 /$ monderusse. 12081

ISSN : $1777-5388$

\section{Éditeur}

Éditions de l'EHESS

\section{Édition imprimée}

Date de publication : 1 juillet 2020

Pagination : $504-508$

ISBN : $978-2-7132-2832-2$

ISSN : $1252-6576$

Référence électronique

Aleksandr Lavrov, « Jan HENNINGS, Russia and Courtly. Europe Ritual and the Culture of Diplomacy,

1648-1725 », Cahiers du monde russe [En ligne], 61/3-4 | 2020, mis en ligne le 01 juillet 2020, consulté le

15 septembre 2022. URL : http://journals.openedition.org/monderusse/12081 ; DOI : https://doi.org/

10.4000/monderusse. 12081 


\section{Jan HENNINGS \\ Russia and Courtly Europe Ritual and the Culture of Diplomacy, 1648-1725 \\ Cambridge : Cambridge university Press (New studies in European history), 2016, $309 \mathrm{p}$.}

La monographie de Jan Hennings résulte de l'étude minutieuse d'un corpus de sources, des rapports de mission (statejnye spiski) des diplomates moscovites à l'étranger, dont la majorité n'est pas publiée. Ces rapports, qui décrivent de façon détaillée les pourparlers diplomatiques et les cérémonies liées aux séjours des missions moscovites à l'étranger ${ }^{1}$, sont normalement étudiés dans le cadre de l'étude des relations bilatérales entre la Moscovie et les puissances étrangères. Quant au cérémonial diplomatique, il n'a attiré l'attention des historiens que dans des cas isolés ${ }^{2}$. Pour son étude, l'auteur a choisi les rapports portant sur les relations de la Moscovie avec l'Europe occidentale. Un choix que le lecteur ne peut que saluer : la prise en compte des rapports écrits par les diplomates moscovites sur leurs séjours dans l'Empire Ottoman, dans le Khanat de Crimée et en Iran aurait exigé plusieurs volumes.

La méthodologie de l'historien est largement liée à celle de l'école de Barbara Stollberg-Rilinger (co-directrice de la thèse soutenue par l'auteur) et à ses travaux sur la « communication symbolique », au sein de laquelle les rituels occupent une place à part ${ }^{3}$. S'appuyant sur les définitions déjà en cours au début des Temps modernes, l'auteur définit la spécificité du cérémonial diplomatique : ses éléments sont « juridiquement contraignants (legally binding) ». Le célèbre livre de Gabriele Scheidegger, postulant une mécompréhension réciproque entre les Moscovites et les Occidentaux, peut constituer un autre repère historiographique de l'auteur ${ }^{4}$. La relation de l'auteur avec la pensée de Scheidegger est presque antagonique. Tandis que l'historienne essaie de décrire et d'interpréter ces mécompréhensions, Hennings affirme que les diplomates moscovites et leurs collègues occidentaux utilisaient le même langage symbolique. Par conséquent, ils se comprenaient et leurs querelles régulières n'étaient pas imputables aux particularités intraduisibles des deux cultures, mais aux conflits réels qui prenaient forme de querelles sur les détails du cérémonial. 
Il est particulièrement intéressant que Hennings n'essaie pas de relativiser les philippiques occidentales sur la « barbarie » des Moscovites en leur opposant un catalogue des préjugés que les Moscovites attribuent aux Occidentaux, comme le fait Scheidegger. Au contraire, il tente de montrer qu'en cas d'échec, ces philippiques s'articulaient autour de l'incapacité du diplomate à atteindre ses objectifs, autant symboliques que réels. Les diplomates occidentaux expliquaient alors le résultat infructueux de leurs entretiens par l'inaptitude des Moscovites à négocier et par leur obstination à respecter scrupuleusement le cérémonial, ce que l'auteur illustre avec l'exemple de la relation de Guy Miège sur l'ambassade de Carlisle.

Arrivé à ce point, il est légitime de s'interroger sur l'existence d'une spécificité moscovite. La diplomatie moscovite était-elle différente de celle des autres États contemporains ? La réponse de l'auteur est double. Premièrement, tandis que les diplomates occidentaux entretenaient normalement une correspondance avec leurs cours, qui leur permettait d'éclaircir tous les problèmes, les Moscovites, du fait de leur éloignement, étaient livrés à eux-mêmes. De là, le caractère détaillé de l'instruction donnée au départ, que les diplomates moscovites devaient soigneusement observer. Deuxièmement, tandis que les diplomates occidentaux citaient une documentation hétérogène pendant les querelles de cérémonial, les Moscovites ne se référaient qu'à leurs propres documents.

Le premier chapitre («Barbarous Ceremonies? Russia’s Places in Early Modern Diplomacy ») porte sur l'image de la Moscovie et de ses ambitions internationales dans les ouvrages occidentaux contemporains. L'auteur commence par un aperçu des opinions de voyageurs et de diplomates, qui se demandaient si la Moscovie appartenait au système européen et répondaient le plus souvent par la négative ${ }^{5}$. Cet aperçu peut paraître trop long, si l'on sait que l'évolution de ces représentations est bien étudiée par Michel Mervaud, Jean-Claude Roberti et Marshall Poe. En revanche, l'analyse de traités sur la diplomatie et le cérémonial diplomatique, parus au XVII ${ }^{\mathrm{e}}$ et au XVIII ${ }^{\mathrm{e}}$ siècle, offre plusieurs éléments nouveaux. Ainsi, les traités sur la hiérarchie des puissances européennes signés par Balthasar Sigismund von Stosch (1677) et par Zacharias Zwantzig (1706) prouvent que les auteurs comprenaient les ambitions impériales des Moscovites, qui employaient le terme «tsar », ainsi que les conséquences du paiement du tribut au khan de Crimée pour les positions internationales de la Moscovie (p. 55). L'auteur attire l'attention sur l'idée très intéressante, présentée par Gottfried Stieve (1715), selon laquelle la Moscovie ne pouvait pas avoir une place fixe dans l'ordre des préséances établi par la curie romaine, car elle n'était pas catholique. Faisant partie du monde orthodoxe, elle occupait la première place au sein de ce dernier (p. 56-57).

Dans le deuxième chapitre («Facts and Fictions : The Organisation of Diplomatic Practice »), l'auteur étudie les institutions moscovites en charge du cérémonial, le Secrétariat des ambassades (Posol'skij prikaz) en particulier, qui produisaient et systématisaient le savoir sur ce sujet. L'auteur établit que la mise en place des cérémonies fut le plus souvent confiée aux pristavy, qui jouaient le même rôle que les introducteurs (conducteurs) des ambassadeurs en France et en Espagne ou les « masters of the cérémonies » en Angleterre (p. 78). Il n'est pas étonnant 
qu'une fonction spéciale, celle de maître de cérémonie, apparaisse dans les temps pétroviens, ni que celle-ci soit occupée par un Italien.

Au premier coup d'œil, on pourrait accepter que la triple hiérarchie moscovite - posol, poslannik et gonec - se traduise sans problèmes et corresponde à la hiérarchie européenne (ambassadeur, envoyé et courrier). La question est complexe. Hennings cite l'approche occidentale de l'époque, selon laquelle « only ambassadors 'cum caractere' conveyed the impression that their principle was present in person, enjoying the prerogatives and privileges due to their master ». En même temps, outre les ambassadeurs, des envoyés et des courriers moscovites réclamaient la même qualité, c'est-à-dire, « d'incarner et de démontrer la dignité souveraine du tsar, qui n'était réservée en Occident qu'au premier rang » (« All Russian diplomats claimed to embody and demonstrate the sovereign dignity of the tsar, which, in the West, was reserved to those of the first rank - p. 104) ». Quand on examine les exemples choisis par Hennings, on peut se demander si un envoyé moscovite qui essaie de s'attribuer des honneurs réservés à un ambassadeur ou des courriers moscovites qui insistent pour obtenir une audience chez l'empereur agissent vraiment au nom du prestige de la Moscovie ou s'ils ne tentent pas plutôt d'élever leur propre statut, ce qui pouvait être important pour leurs familles dans les querelles de préséances (mestničeskie spory). Nonobstant ces différences, l'auteur pointe la similarité essentielle du service diplomatique moscovite avec ses homologues occidentaux de l'époque.

Le troisième chapitre est consacré aux relations entre la Moscovie et l'Angleterre dans le troisième quart du XVII ${ }^{\mathrm{e}}$ siècle («Through the Prism of Ritual. AngoRussian Encounters in the Seventeenth Century »). La version traditionnelle de ces relations est simple : le tsar Aleksej Mihajlovič prend prétexte de l'exécution du roi Charles I pour abolir les privilèges exclusifs du Muscovite Company. Pourtant, dans l'esprit de la Realpolitik, les diplomates d'Oliver Cromwell furent accueillis à Moscou. En vrai légitimiste, le tsar est heureux de voir la monarchie rétablie, mais il ne restaure pas les privilèges. L'analyse du cérémonial permet à l'auteur de nuancer cette image, traditionnelle dans l'historiographie. Tout d'abord, il montre que les Moscovites introduisent de petits détails dans le cérémonial qui marquent le statut inférieur du diplomate de Cromwell. Ensuite, l'auteur réussit à montrer le vrai bilan de la mission de Charles Howard, premier comte de Carlisle, (16631664) qui ne parvint pas à renégocier les privilèges. Cependant, les Moscovites lui réservèrent un cérémonial très digne, copié sur celui avec lequel l'ambassade moscovite avait été accueillie à Londres, mais qui ne prémunit pas des querelles de cérémonial.

Le quatrième chapitre porte sur la présence de la Grande Ambassade de Pierre le Grand à Vienne (« Stage and Audience. The Grand Embassy to Vienna (1698) and Peter I's Visit to Paris (1717) »). Les historiens prêtent normalement attention à la rencontre privée qui se tint entre l'empereur et le tsar, venu à Vienne incognito. L'auteur observe très justement, pointant le caractère innovateur de la diplomatie pétrovienne, que les rencontres de monarques étaient rares à cette époque. Cette remarque est particulièrement importante pour le lecteur contemporain, qui ne se 
représente plus la diplomatie comme une négociation de ministres, mais comme une série de rencontres entre leaders. Cependant, l'auteur demande de ne pas sousestimer le côté cérémoniel de l'accueil de la Grande Ambassade. Hennings montre que les Impériaux voulaient répéter les cérémonies mises en place pour l'accueil de l'ambassade moscovite en 1687, tandis que les Moscovites désiraient y ajouter des honneurs, qui leur furent refusés par les Impériaux, qui craignaient que ces innovations pussent être considérées comme un précédent par les diplomates des autres puissances.

Le dernier chapitre est consacré aux changements introduits par Pierre le Grand («From Insult to Imperator. Changes and Continuities in the Reign of Peter I »). De prime abord, on pourrait parler d'un renversement des rôles. En effet, si, auparavant les diplomates occidentaux déploraient l'obsession moscovite pour les cérémonies, désormais, le tsar, méprisant les cérémonies, semblait rendre aux Occidentaux la monnaie de leur pièce. Ici l'auteur prête une attention majeure à l'attitude personnelle du tsar et à son engagement militaire, qui ne permettait pas de mettre en place des cérémonies. Pourtant, derrière ces deux facteurs se dessine un phénomène beaucoup plus important, celui de la dissolution de la cour moscovite, qui était le cadre de toutes les cérémonies, y compris des accueils d'ambassadeurs. L'auteur prouve que la rupture avec le passé n'a pas été radicale. Les diplomates de Pierre le Grand étaient conscients du rôle des cérémonies, et, désormais, ils multiplient leurs références, ils s'appuient sur les archives diplomatiques moscovites, mais aussi sur les traités occidentaux traduits en russe. De plus, le rapport final (statejnyj spisok), élément clé du savoir diplomatique moscovite, disparaît peu à peu, du fait du caractère beaucoup plus mobile et intense du service diplomatique. Alors que, selon Hennings, la création du réseau de représentations permanentes russes à l'étranger réaffirme l'importance du cérémonial.

Le livre de Jan Hennings est plus qu'un enrichissement, il constitue une percée vers une nouvelle problématique. Toutefois, il peut faire l'objet de plusieurs critiques. La première porte sur le choix des exemples. Les relations avec l'Angleterre, largement marquées par la problématique commerciale, n'étaient pas vitales pour la Moscovie. En revanche, on trouve très peu d'éléments sur le cérémonial dans les relations avec la Pologne-Lituanie, un voisin dont le rôle dans la politique internationale de la Moscovie ne peut pas être sous-estimé. L'absence d'un chapitre consacré aux diplomates moscovites à Versailles est d'autant plus regrettable que l'auteur prouve par quelques remarques précises que le cérémonial jouissait là d'une place inédite.

Ensuite, il est difficile de comprendre pourquoi la Déclaration du tsar Aleksej Mihajlovič est citée comme un document diplomatique, quand il s'agit plutôt d'une mystification royaliste ${ }^{6}$. Ce document expose plusieurs idées inédites pour la diplomatie moscovite, dont l'appel à la formation de la coalition des monarques européens contre l'Angleterre (« League between the Kingdoms », (p. 119, 120)). La question de savoir dans quelle mesure les idées de la Déclaration pouvaient être connues du tsar ou partagées par lui, mérite une étude à part. Enfin, il reste à déplorer l'absence de sources visuelles, notamment, et pour n'en citer que deux, 
la célèbre gravure de Juan Carreño de Miranda montrant l'ambassade moscovite à Ratisbonne (Prado) et les portraits de Petr Ivanovič Potemkin (Ermitage), dus à Godfrey Kneller.

1 - Les diplomates moscovites recevaient avant leur départ une instruction (nakaz), avec la description détaillée des cérémonies prévues pour leur mission. Le bilan réel de la mission, y compris les cérémonies, était l'objet d'un rapport de mission que les diplomates établissaient après leur retour à Moscou. Les rapports de mission furent souvent intégrés aux registres des ambassades (posol'skie knigi), qui réunissaient tous les documents importants relatifs aux relations avec un État étranger.

2 - Il faut noter une exception heureuse pour l'historiographie soviétique : le livre de l'historien et écrivain Leonid A. Juzefovič, destiné au grand public, Kak v posol'skih obyčajah vedetsja, M. : Meždunarodnye otnošenija, 1988.

3 - Voir la traduction française : B. Stollberg-Rilinger, Les vieux habits de l'Empereur. Une histoire culturelle des institutions du Saint-Empire à l'époque moderne, P., 2013.

4 - G. Scheidegger, Perverses Abendland-barbarisches Russland: Begegnungen des 16. und 17. Jahrhunderts im Schatten kultureller Missverständnisse, Zürich, 1993.

5 - Une source formidable, trouvée par l'auteur, montre toutes les difficultés de l'interprétation. Il s'agit du privilège de Louis XIV, donné à Antoine Benoist, qui fut autorisé à fabriquer des figures en cire des « ambassadeurs de Siam, Moscovie, Maroc, Alger, doge de Gennes, Cour du Grand Seigneur et autres cours étrangères ». « Clearly, Russia’s place on the map of cultural representation was near non-European societies like those of Siam, Morocco, or the Ottoman Empire », résume Hennings (p. 36) ; voir l'original français du privilège : Nouvelles archives de l'art français (troisième série, Vol. VI), P., 1890, p. 167). Pourtant, la présence dans cette liste du doge de Gênes met en cause toute l'interprétation de l'historien - ce dernier était catholique et ne portait pas d'habits exotiques, mais il était ennemi et captif de Louis XIV.

6 - Z.I. Roginskij « Tak nazyvaemyj "Protest carja Alekseja Mihajloviča po povodu kazni korolja Karla I [Ladite « protestation du tsar Aleksej Mihajlovič à propos de l'exécution du roi Charles I"]», Učenye zapiski Jaroslavskogo pedagogičeskogo instituta, vyp. XXII, 1957, p. 287-308.

\author{
Aleksandr Lavrov \\ Sorbonne Université
}

